\title{
TGFBI promoter hypermethylation correlating with paclitaxel chemoresistance in ovarian cancer
}

\author{
Ning Wang ${ }^{1,2}$, Hui Zhang ${ }^{1}$, Qin Yao ${ }^{2}$, Yankui Wang ${ }^{2}$, Shuzhen Dai ${ }^{2}$ and Xingsheng Yang ${ }^{1 *}$
}

\begin{abstract}
The purpose of this study is to determine the methylation status of Transforming growth factor-beta-inducible gene-h3 (TGFBI) and its correlation with paclitaxel chemoresistance in ovarian cancer. The methylation status of TGFBI was examined in ovarian cancer and control groups by methylation-specific PCR (MSP) and bisulfite sequencing PCR (BSP). The TGFBI expression and cell viability were compared by Quantitative Real-Time PCR, Western Blotting and MTT assay before and after demethylating agent 5-aza-2'-deoxycytidine (5-aza-dc) treatment in 6 cell lines (SKOV3, SKOV3/TR, SKOV3/DDP, A2780, 2780/TR, OVCAR8). In our results, TGFBI methylation was detected in 29/40 (72.5\%) of ovarian cancer and 1/10 (10\%) of benign ovarian tumors. No methylation was detected in normal ovarian tissues $(P<0.001)$. No statistical correlation between RUNX3 methylation and clinicopathological characteristics was observed. A significant correlation between TGFBI methylation and loss of TGFBI mRNA expression was found $(P<0.001)$. The methylation level of TGFBI was significantly higher in paclitaxel resistant cell lines (SKOV3/TR and 2780/TR) than that in the sensitive pairs $(P<0.001)$. After 5 -aza-dc treatment, the relative expression of TGFBI mRNA and protein increased significantly in SKOV3/TR and A2780/TR cells. However, no statistical differences of relative TGFBI mRNA expression and protein were found in other cells (all $P>0.05$ ), which showed that re-expression of TGFBI could reverse paclitaxel chemoresistance. Our results show that TGFBI is frequently methylated and associated with paclitaxel-resistance in ovarian cancer. TGFBI might be a potential therapeutic target for the enhancement of responses to chemotherapy in ovarian cancer patients.
\end{abstract}

Keywords: Ovarian cancer, transforming growth factor-beta-inducible gene-h3, methylation, chemoresistance, paclitaxel

\section{Introduction}

Epithelial ovarian cancer is the most lethal gynecologic malignancy, with 21990 estimated new cases and 15 460 deaths in the USA in 2011 [1]. Reasons for this high lethality include the advanced stage at which patients are diagnosed and the inherent aggressive biology of this cancer.

Maximal surgical cytoreduction followed by systemic chemotherapy with carboplatin and paclitaxel is the current standard treatment modality for advanced ovarian cancer [2]. A key feature of ovarian cancer is its sensitivity to chemotherapeutic drugs such as paclitaxel, a prototype taxane, stabilizes microtubule polymers leading to mitotic arrest and apoptosis [3]. Unfortunately, ovarian

\footnotetext{
* Correspondence: xingshengyang@yahoo.com

'Department of Obstetrics and Gynecology, Qilu Hospital, Shandong

University, 107 Wenhuaxi Road, Jinan 250012, P.R. China

Full list of author information is available at the end of the article
}

cancer cells, with their unstable genomes [4], are initially sensitive to these drugs, but long term utilization may result in the chemoresistance [5].

Epigenetic alterations play an important role in the initiation and progression of cancer [6-8]. Hypermethylation of $\mathrm{CpG}$ rich islands in promoter regions of genes has been characterized as a common epigenetic alteration for the silencing or inactivation of tumor suppressor genes and transcriptional repression in human malignancies [9,10], including ovarian cancer [11-13]. In recent years, emerging evidence has also linked epigenetic changes to the development of drug resistance $[14,15]$.

Transforming growth factor-beta-inducible gene-h3 (TGFBI) is a secreted protein first identified in a human lung adenocarcinoma cell line treated with transforming growth factor- $\beta$ [16]. It has been shown to possess tumor suppressor function in vitro studies $[17,18]$, and

\section{() Biomed Central}

(c) 2012 Wang et al; licensee BioMed Central Ltd. This is an Open Access article distributed under the terms of the Creative Commons Attribution License (http://creativecommons.org/licenses/by/2.0), which permits unrestricted use, distribution, and reproduction in any medium, provided the original work is properly cited. 
to be correlated with specific sensitization to paclitaxel by inducing stabilization of microtubules via integrinmediated signaling pathways [19]. Recently, promoter hypermethylation of TGFBI was found in lung $[20,21]$ and prostate cancer [20]. However, the role of TGFBI methylation in paclitaxel chemoresistance in ovarian cancer is unknown. Therefore, a better understanding of this epigenetic mechanism of TGFBI in ovarian cancer could facilitate the generation of new drugs that re-sensitize tumor cells to paclitaxel [4].

In this study, we examined the methylation status and expression of TGFBI in epithelial ovarian cancer tissues, paclitaxel-sensitive and -resistant ovarian cancer cell lines in order to determine whether the methylation of TGFBI is asscociated with paclitaxel chemoresistance.

\section{Materials and methods}

Ovarian cancer tissue samples and cell lines

From April 2008 to April 2009, 40 primary epithelial ovarian cancer(, 10 benign tumor and 10 normal ovarian tissues) were collected at the Department of Obstetrics and Gynecology, The Affiliated Hospital of Medical College, Qingdao University, China. The mean age of the patients was 43 years (range 21-77 years). The ovarian cancer patients have different histological types: serous papillary carcinoma $(\mathrm{n}=20)$, mucinous carcinoma $(\mathrm{n}=$ 13), endometrioid carcinoma $(n=7)$. Six patients were in stage I, ten patients were in stage II, twenty-four patients were in stage III. Twenty-two patients had metastasis to pelvic lymph nodes. Eleven tumors were well-moderately differentiated, and 29 tumors were poorly differentiated. Ten benign tumor and 10 normal ovarian tissues were collected as control. All samples were obtained prior to chemotherapy or radiation therapy, which were placed in liquid nitrogen immediately after resection and stored at $-80^{\circ} \mathrm{C}$ until use. The malignant and normal diagnosis was performed by pathologists. The study was performed after approval by our institute Medical Ethics Committee.
Human SKOV3, A2780 and OVCAR8 ovarian cancer cell lines were obtained from the bioengineering centre of The Affiliated Hospital of Medical College, Qingdao University, China. The chemoresistant cell lines (SKOV3/ DDP, SKOV3/TR, and A2780/TR) were purchased from the China Center for Type Culture Collection (Wuhan, China). These cells were maintained in DMEM with $10 \%$ fetal bovine serum and $100 \mathrm{U} / \mathrm{ml}$ penicillin/streptomycin at $37^{\circ} \mathrm{C}$. SKOV3/TR and A2780/TR were cultured in RPMI-1640 medium containing $0.3 \mu \mathrm{mol} / \mathrm{L}$ paclitaxel to maintain the drugresistant phenotype.

Cells were grown to $70 \%$ confluence and treated with $10 \mu \mathrm{mol} / \mathrm{L}$ of demethylating agent (5-aza-2'-deoxycytidine, 5-aza-dc) (Sigma-Aldrich, St. Louis, MO, USA) for 3 days [22]. After the treatment, cells were harvested and extracted for DNA, RNA and protein.

\section{Nucleic acid isolation}

The EZNA Tissue DNA Kit (Omega Corp, USA) was used to extract high purity DNA from different ovarian tissues and ovarian cancer cell lines. Total DNA content was quantified by UV absorbance value measured at A260 and A280, and diluted to a concentration of $1 \mu \mathrm{g} / 100 \mu \mathrm{l}$.

\section{Methylation-specific PCR (MSP) and bisulfite sequencing PCR (BSP)}

DNA from tissue samples and cell lines were subjected to bisulfite treatment using CpGgenome DNA Modification Kit (Chemicon, USA). Sequences, Tm, and product length of each primer used for MSP and BSP analysis are summarized in Table 1 The band expanded with methylation-specific PCR primers corresponding to the DNA methylation in the promoter region was marked as "M". The band expanded with non-methylation-specific primers was marked as " $U$ ".

\section{Quantitative real-time PCR (qRT-PCR)}

Total RNA was extracted from cells with Trizol reagent (Invitrogen, San Diego, CA, USA), and it was reverse

Table 1 Sequences of Primers for MSP and RT-PCR

\begin{tabular}{|c|c|c|c|c|}
\hline Gene & Primer & Sequences & Temp $\left({ }^{\circ} \mathrm{C}\right)$ & Length(bp) \\
\hline \multirow[t]{8}{*}{ TGFBI } & M-F & 5'-gAAAATTGAGTACGGGTATAGTGC-3' & 58 & 162 \\
\hline & $M-R$ & $5^{\prime}$-CCAAATTAAATAAACTACGAACGAA-3' & & \\
\hline & $\mathrm{U}-\mathrm{F}$ & 5'-AAAATTGAGTATGGGTATAGTGTGG-3' & 56 & 161 \\
\hline & U-R & 5'-CCAAATTAAATAAACTACAAACAAA-3' & & \\
\hline & $B-F$ & 5'-TGGAAGTAGTTATAGGAGGTTAAAGG-3' & 55 & 402 \\
\hline & $B-R$ & $5^{\prime}-$ CCCAAAACCAAAACCAAAAC-3' & & \\
\hline & qRT-F & 5'-GGCTGCAGAGTCTGATGTGT-3' & 55 & 81 \\
\hline & qRT-F & 5'-CGCTCACTTCCAGAGAGATG-3' & & \\
\hline \multirow[t]{2}{*}{ GAPDH } & qRT-F & 5'-GGACCTGACCTGCCGTCTAG-3' & 55 & 99 \\
\hline & qRT-R & 5'-TAGCCCAGGATGCCCTTGAG-3' & & \\
\hline
\end{tabular}

M: methylated primers [23], U: unmethylated primers [23], B: primers of BSP primers (designed using MethPrimer), qRT, primers of Quantitative Real-Time PCR. 
transcribed using miScript Reverse Transcription Kit (Qiagen, Hilden, Germany). The primers for mRNA are listed in Table 1. The quantification was performed with QuantiTect Probe RT-PCR (Qiagen, Hilden, Germany). The comparative threshold cycle method was used to determine gene relative expression.

\section{Western blotting}

Cells were washed twice with ice-cold phosphate-buffered saline and lysed using a modified RIPA buffer supplemented with $1 \mathrm{mM}$ PMSF. The protein concentration was detected using $\mathrm{BCA}$ protein assay (Pierce, Rockford, IL, USA). Proteins were loaded onto $10 \%$ and 5\% SDS-PAGE and electrophoretically transferred to a PVDF membrane (Millipore, Bedford, MA, USA). After blocking with $5 \%$ non-fat milk in PBSTween 20 for $2 \mathrm{~h}$ at room temperature, the membranes were incubated with anti-human monoclonal $\beta$-actin and anti-human TGFBI primary antibody overnight at $4^{\circ} \mathrm{C}$. Horseradish peroxidase-conjugated secondary antibody was added for $2 \mathrm{~h}$ at room temperature. The Detection was performed by chemiluminescence.

\section{MTT assay}

MTT Cell Proliferation Assay (Biosharp, USA) was used to measure cell viability. Before and after treated with 5aza-dc, $1 \times 10^{4}$ cells/well were seeded in 96-well plates containing complete medium and incubated for $24 \mathrm{~h}$. Then cells were exposed to serial dilutions of paclitaxel in a total volume of $200 \mu \mathrm{L}$ in four replicate wells. After 48 hours, plates were added $20 \mu \mathrm{l}$ of MTT reagent and incubated for $4 \mathrm{~h}$, and then formazane crystals formed were dissolved in $150 \mu \mathrm{l}$ of dimethyl sulfoxide (Wako, Tokyo, Japan). The optical density was measured at 490 $\mathrm{nm}$ on a microplate reader. The half maximal inhibitory concentration (IC50) value was assessed by different concentrations of paclitaxel (0.01, 0.1 and $1 \mu \mathrm{M})$.

\section{Statistical analyses}

All statistical analyses were performed using SPSS 15.0. Fisher's exact test or and $\chi^{2}$ test were used to compare TGFBI methylation status among cases and between various clinicopathologic variables. Pearson correlation analysis was used to evaluate the relationship between TGFBI methylation status and mRNA expression. The differences of TGFBI mRNA and protein expression before and after 5 -aza-dc treatment were analyzed by the Paired-Samples $t$ test. $P<0.05$ was considered statistically significant.

\section{Results}

Frequency of TGFBI methylation in ovarian cancer tissues We determined the frequency of TGFBI methylation in 40 primary ovarian cancer samples, 10 benign ovarian tumors and 10 normal ovarian tissues by MSP (Figure 1). TGFBI in all of the normal ovarian tissues was completely methylation free, which showed success of DNA modification. The overall frequency of methylation in benign ovarian tumors was $10.0 \%(1 / 10)$. For ovarian cancer tissues, $72.5 \%(29 / 40)$ of methylation was observed. The data demonstrated that the difference of TGFBI methylation frequency among ovarian cancers, benign ovarian tumors and normal ovarian tissues was statistically significant $(P<0.001)$.

The methylation status of the ovarian cancers was compared with clinicopathological characteristics from these patients including age, histological type, tumor stage, histological grade and lymphatic metastasis. No significant correlation between TGFBI methylation and any of these parameters was observed for the ovarian cancer patients (Table 2).

\section{Expression of TGFBI mRNA in ovarian cancer tissues}

To examine whether TGFBI methylation results in the suppression of TGFBI expression, we examined TGFBI mRNA expression by qRT-PCR in 40 ovarian cancer tissues and 10 normal ovarian tissues. TGFBI mRNA expression was detected in all the normal ovarian tissues (10/10) and in most of the unmethylated ovarian cancer tissues (10/11). In contrast, TGFBI expression was not detected in the TGFBI-methylated ovarian cancer tissues (27/29), except for 2 tissues. We compared the TGFBI mRNA expression results of these ovarian cancer tissues with the TGFBI methylation data and found a significant correlation between TGFBI methylation and loss of TGFBI

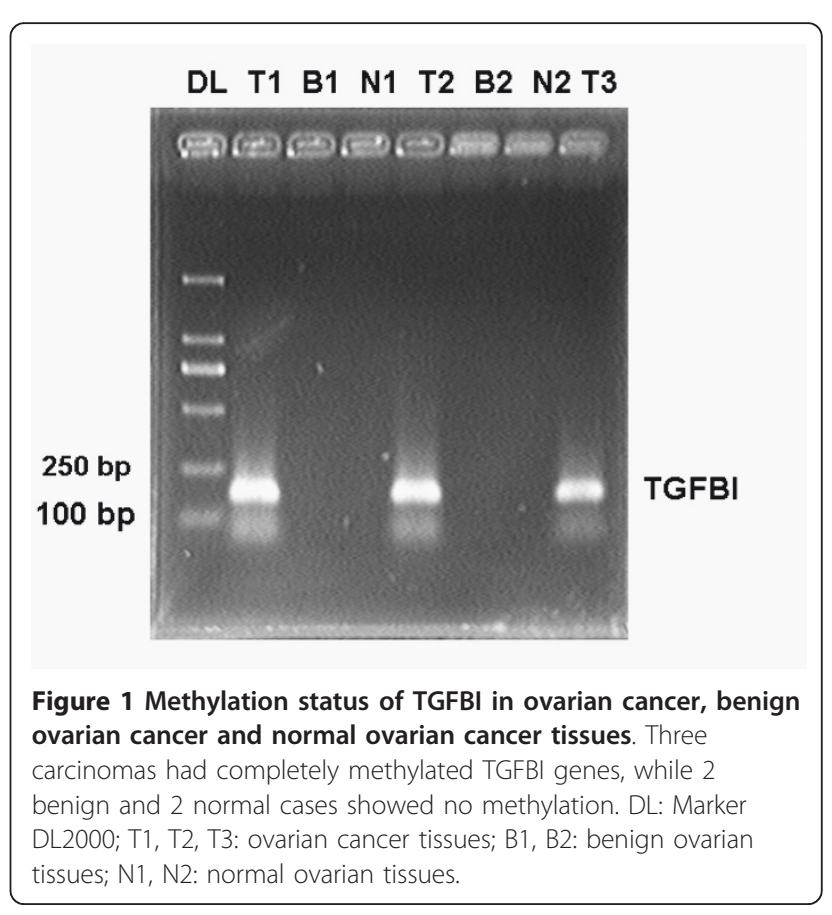


Table 2 Association of TGFBI methylation and clinicopathologic variables in $\mathbf{4 0}$ ovarian cancer patients

\begin{tabular}{llll}
\hline $\begin{array}{l}\text { Clinicopathologic } \\
\text { characteristics }\end{array}$ & $\begin{array}{l}\text { Number } \\
(\mathbf{n})\end{array}$ & $\begin{array}{l}\text { Methylation } \\
(\mathbf{\%})\end{array}$ & $\begin{array}{c}\boldsymbol{P} \\
\text { value }\end{array}$ \\
\hline Age at diagnosis & 14 & $9(64.3)$ & 0.3932 \\
$<50$ years & 26 & $20(76.9)$ & \\
$\begin{array}{l}\text { 250 years } \\
\text { Histological type }\end{array}$ & 20 & $16(80.0)$ & 0.4814 \\
Serous adenocarcinoma & 13 & $9(69.2)$ & \\
Mucinous adenocarcinoma & 7 & $4(57.1)$ & \\
Endometrioid adenocarcinoma & 7 & & \\
Tumor stage & & $2(33.3)$ & 0.0661 \\
I & 6 & $8(80.0)$ & \\
II & 10 & $19(79.2)$ & \\
III & 24 & & \\
Histological grade & & $2(40.0)$ & 0.5532 \\
G1 & 4 & $5(71.4)$ & \\
G2 & 7 & $22(75.9)$ & \\
G3 & 29 & $13(72.2)$ & 0.9716 \\
Lymphatic metastasis & & $16(72.7)$ & \\
No & 18 & & \\
Yes & 22 & & \\
\hline
\end{tabular}

mRNA expression $(P<0.001)$. These results suggest that the inactivation of TGFBI expression is closely correlated with gene methylation in ovarian cancer tissues.

\section{Demethylation and re-expression of TGFBI after treating} with 5 -aza-dc in ovarian cancer lines

We detected the methylation status of TGFBI promoter region in 4 ovarian cell lines by MSP and BSP before and after treating with 5-aza-dc. Before treatment, there was partial TGFBI methylation detected in SKOV3 and A2780 cells (42.9\% and $35.2 \%$ of total CpG sites, respectively). In contrast, almost complete hypermethylation were found in SKOV3/TR (94.3\% of total CpG sites) and A2780/TR (91.4\% of total CpG sites) cells (Figure 2).

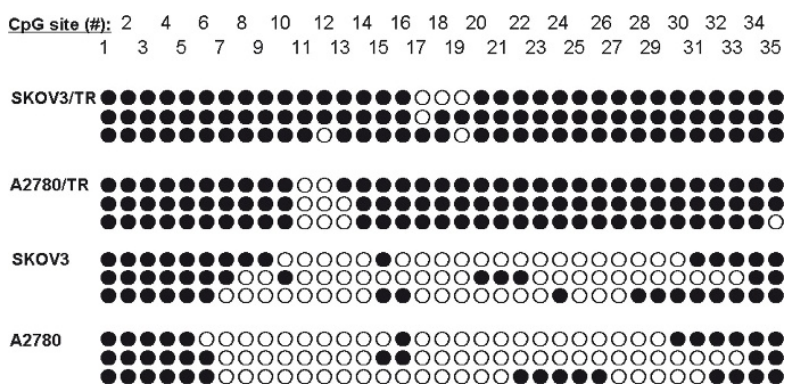

Figure 2 Bisulfite sequencing of SKOV3, SKOV3/TR, A2780 and A2780/TR. Paclitaxel-resistant cell lines (SKOV3/TR and A2780/TR) showed almost complete CpG methylation (91.4\% and $97.1 \%$ of total CpG sites, respectively), the sensitive cell lines SKOV3 and A2780 showed partial methylation of CpG islands (42.9\% and 35.24\% of total CpG sites, respectively)
After 5-aza-dc treatment, a " $U$ " band appeared while the "M" band did not disappear in A2780/TR cell line, which demonstrated that the methylation was partially reversed. In another cell line SKOV3/TR, the "M" band disappeared and only a " $U$ " band was left, indicating that the methylation had been completely reversed (Figure 3).

The expression of TGFBI mRNA was examined in all the 6 ovarian cancer cell lines by qRT-PCR before and after treating with 5-aza-dc (Figure 4). Our data showed that the relative expression of TGFBI mRNA increased significantly after treating with 5 -aza-dc in SKOV3/TR $(7.8 \pm 0.9$ vs. $0, P<0.001)$ and A2780/TR $(6.4 \pm 0.2$ vs.0, $P<0.001)$ cells. However, no statistical differences of relative TGFBI mRNA expression were found after 5 aza-dc administration in OVCAR8 (1.6 \pm 0.3 vs. $0.8 \pm$ $0.1, P>0.05)$, $\operatorname{SKOV} 3(5.1 \pm 0.2$ vs. $4.2 \pm 0.2, P>0.05)$, SKOV3/DDP $(1.4 \pm 0.9$ vs. $0.9 \pm 0.2, P>0.05)$ and A2780 cells $2.7 \pm 0.9$ vs. $2.1 \pm 0.7, P>0.05$ ).

In addition, we examined TGFBI protein (TGFBIp) expression in all the cell lines by Western blotting (Figure 5). The data showed that the expression of TGFBIp in SKOV3/TR and A2780/TR cell lines was statistically up-regulated after 5 -aza-dc administration $(P<0.01$ and $P<0.01$, respectively). By contrast, no significant differences were found in other cell lines (all $P>0.05$ ), which was coincident with the results of qRT-PCR.

\section{MTT assay}

Further, we studied the effect of cell proliferation of 5aza-dc on paclitaxel-resistant cell lines by MTT viability assay. Our results showed that the rate of cell inhibition was significantly increased in SKOV3/TR and A2780/TR than that in control groups at several paclitaxel

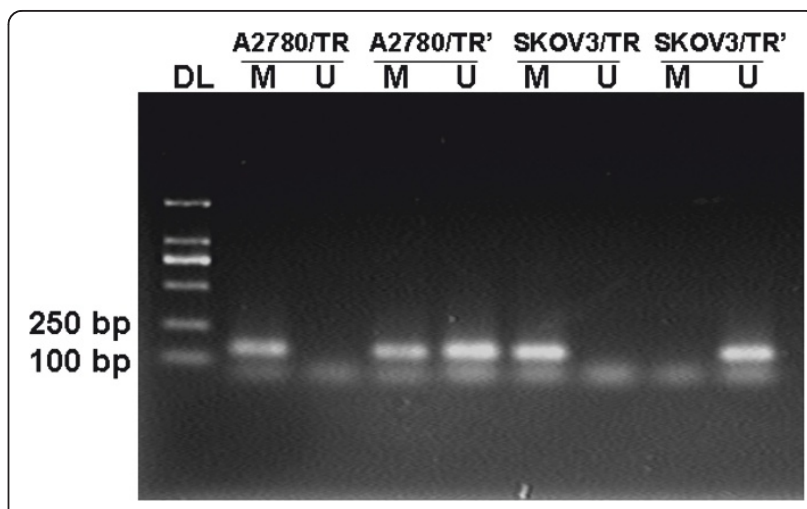

Figure 3 MSP analysis of TGFBI in paplitaxel resistant cell lines after demethylation by $\mathbf{5}$-aza-dc. After 5 -aza-dc treatment, a " $U$ " band appeared while the " $\mathrm{M}$ " band did not disappear in A2780/TR cell line. In another cell line SKOV3/TR, the " $\mathrm{M}$ " band disappeared and only a "U" band was left. DL: Marker DL2000; SKOV3/TR, A2780/ TR: before treatment; SKOV3/TR', A2780/TR': after treatment; U: unmethylation, M: methylation. 


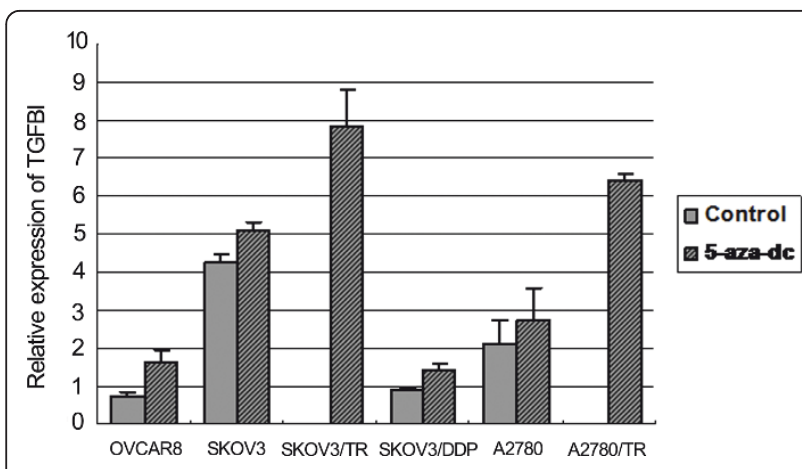

Figure 4 Quantitative real-time RT-PCR analysis of TGFBI expression in ovarian cancer cells. It showed that the relative expression of TGFBI mRNA increased significantly after treating with 5-aza-dc in SKOV3/TR and A2780/TR cells. However, no statistical differences of relative TGFBI mRNA expression were found after 5aza-dc administration in other cell lines.

concentrations of $0.01,0.1$ and $1 \mu \mathrm{M}(P<0.05)$ (Figure 6). The IC50 of SKOV3/TR obviously decreased after 5 aza-dc administration $(0.19 \pm 0.01 \mu \mathrm{M}$ vs. $0.42 \pm 0.02$ $\mu \mathrm{M}, P=0.001)$, which was similar with the results of A2780/TR $(0.012 \pm 0.0001 \mu \mathrm{M}$ vs. $0.33 \pm 0.011 \mu \mathrm{M} ; P=$ 0.001).

\section{Discussion}

In this study, we first detected the methylation status of the 5' CpG island of TGFBI in different ovarian tissues using MSP and BSP in order to determine whether TGFBI inactivation by DNA methylation is characteristic of human ovarian cancer. After repeated experiments, our results showed that the TGFBI is frequently methylated in ovarian cancer. Its methylation can be used as a novel epigenetic biomarker for ovarian cancer detection.

We further measured TGFBI mRNA and protein levels by RT-PCR and IHC in ovarian cancer tissues. Then we compared the TGFBI expression results with the TGFBI methylation data and found a significant inverse correlation between TGFBI methylation and TGFBI expression, which confirmed the important role

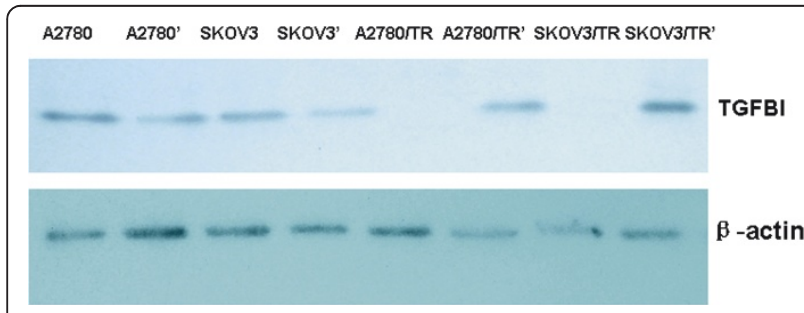

Figure 5 The TGFBIp expression before and after treatment of 5-aza-dc by Western blotting. Expression of TGFBIp in SKOV3/TR and A2780/TR cell lines was sharply up-regulated after treatment of 5-aza-dc. A2780, SKOV3, A2780/TR, SKOV3/TR: before treatment; A2780', SKOV3', A2780/TR', SKOV3/TR': after 5-aza-dc treatment.

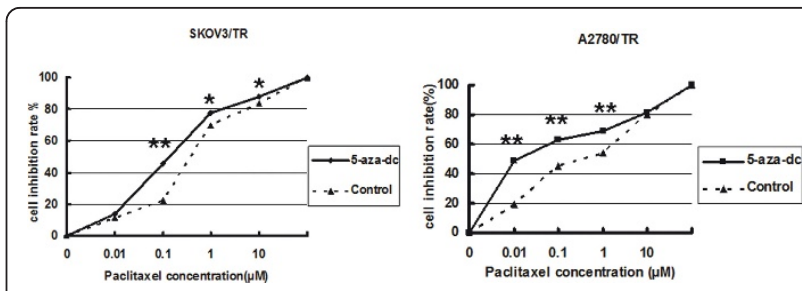

Figure 6 Demethylation of TGFBI restores the sensitivity of paclitaxel-resistant ovarian cells. The inhibition rates in paclitaxelresistant cells with 5 -aza-dc treatment were increased significantly than control ones (* $P<0.05$; ${ }^{* *} P<0.01$ ).

of promoter methylation in regulating TGFBI expression. However, because 1 ovarian cancer tissue lacking TGFBI mRNA expression was not methylated, we presume that mechanisms of inactivating the gene other than methylation must exist.

Recently, Shah et al. [20] reported that TGFBI methylation was associated with tumor recurrence and metastasis, suggesting that TGFBI is required to suppress the aggressiveness of prostate and lung cancer. In our study, the methylation rate of carcinomas with poor differentiation was higher than those with well differentiation. Meanwhile, higher methylation rate was also found in late stage patients with ovarian cancers, though no significant correlation was found between TGFBI methylation status and clinicopathological characteristics, which was in accordance with the results of Kang et al [23]. Our results showed that there were different patterns of mythylation according to the histology and the tumor grade, and revealed that hypermethylation of TGFBI in ovarian cancer might be associated with unfavourable prognosis. Further studies with large sample size and long-term follow-up are required to confirm the hypothesis.

Chemoresistance is the major cause of treatment failure for ovarian cancer. It is reported that DNA methylation may act as a potential cause of chemotherapy drug resistance [24-26]. In a recently study by $\mathrm{Li}$ et al. [27], cisplatin-sensitive and -resistant ovarian cancer cells were analyzed by methylation and mRNA expression microarray. Their results revealed that DNA hypermethylation may contribute to the onset of the chemoresistance in ovarian cancer.

In our study on cell lines, almost complete methylation pattern of the TGFBI promoter in 2 paclitaxelresistant cell lines (SKOV3/TR and A2780/TR) was observed, with a complete loss or low level of TGFBI expression in these cell lines. In contrast, only sparsely methylated or unmethylated CpG sites were identified in cell lines with a rich level of TGFBI expression, including SKOV3, A2780, OVCAR8, and SKOV3/DDP ovarian cancer cell lines. Our results identified strong relation between TGFBI expression and response to 
chemotherapy. To our knowledge, this is the first evidence of TGFBI hypermethylation as a mechanism of paclitaxel chemoresistance in ovarian cancer.

Further, our results were confirmed by using DNA methylation inhibitors. The relative expression of TGFBI mRNA and protein increased significantly after treating with 5-aza-dc in palitaxel-resistant cells. However, no statistical differences of TGFBI expression were found after 5 -aza-dc administration in other 4 cell lines. In addition, MTT assay showed that the rate of cell inhibition was significantly increased in SKOV3/TR and A2780/TR after 5-aza-dc treatment, which suggested that chemotherapy sensitivity to paclitaxel was enhanced and chemoresistance was reversed.

In conclusion, our study indicated that promoter hypermethylation of TGFBI is a frequent event in ovarian cancer. TGFBI methylation was associated with paclitaxel chemoresistance, and it can be used as a potential epigenetic biomarker and therapeutic target of paclitaxel resistance in ovarian cancer.

\section{Acknowledgements}

This work was supported by grants from National Natural Science Foundation of China (No. 81001167, No. 81172480/H1621, No. 81101973/ H1621).

\section{Author details}

'Department of Obstetrics and Gynecology, Qilu Hospital, Shandong University, 107 Wenhuaxi Road, Jinan 250012, P.R. China. 'Department of Obstetrics and Gynecology, Affiliated Hospital of Medical College, Qingdao University, 16 JiangSu Road, Qingdao 266003, P.R. China.

\section{Authors' contributions}

NW and XSY designed and coordinated the study, carried out data interpretation, and drafted the manuscript; $\mathrm{HZ}$ participated in the conception and design of the study, and participated in drafting of manuscript; QY participated in the design of the study and performed the statistical analysis; SZD and YKW conceived of the study, and participated in its design and coordination and helped to draft the manuscript. All authors read and approved the final manuscript.

\section{Competing interests}

The authors declare that they have no competing interests.

Received: 18 October 2011 Accepted: 16 January 2012

Published: 16 January 2012

\section{References}

1. Siegel R, Ward E, Brawley O, Jemal A: Cancer statistics, 2011: the impact of eliminating socioeconomic and racial disparities on premature cancer deaths. CA Cancer J Clin 2011, 61:212-236.

2. Matei D: Novel agents in ovarian cancer. Expert Opin Investig Drugs 2007, 16:1227-1239.

3. McGuire WP, Hoskins WJ, Brady MF, et al: Cyclophosphamide and cisplatin compared with paclitaxel and cisplatin in patients with stage III and stage IV ovarian cancer. N Engl I Med 1996, 334:1-6.

4. Taniguchi T, Tischkowitz M, Ameziane N, et al: Disruption of the Fanconi anemia-BRCA pathway in cisplatin-sensitive ovarian tumors. Nat Med 2003, 9:568-574

5. Ferrandina G, Zannoni GF, Martinelli E, et al: Class III beta-tubulin overexpression is a marker of poor clinical outcome in advanced ovarian cancer patients. Clin Cancer Res 2006, 12:2774-2779.
6. Yoshikawa H, Matsubara K, Qian GS, et al: SOCS-1, a negative regulator of the JAK/STAT pathway, is silenced by methylation in human hepatocellular carcinoma and shows growth-suppression activity. Nat Genet 2001, 28:29-35.

7. Li QL, Ito K, Sakakura C, et al: Causal relationship between the loss of RUNX3 expression and gastric cancer. Cell 2002, 109:113-124.

8. Momparler RL: Cancer epigenetics. Oncogene 2003, 22:6479-6483.

9. Feinberg AP, Tycko B: The history of cancer epigenetics. Nat Rev Cancer 2004, 4:143-153.

10. Esteller M: Epigenetics in cancer. N Engl J Med 2008, 358:1148-1159.

11. Yoon MS, Suh DS, Choi KU, et al: High-throughput DNA hypermethylation profiling in different ovarian epithelial cancer subtypes using universal bead array. Oncol Rep 2010, 24:917-925.

12. Sellar GC, Watt KP, Rabiasz GJ, et al: OPCML at $11 \mathrm{q} 25$ is epigenetically inactivated and has umor-suppressor function in epithelial ovarian cancer. Nat Genet 2003, 34:337-343.

13. Zhang H, Zhang S, Cui J, Zhang A, Shen L, Yu H: Expression and promoter methylation status of mismatch repair gene hMLH1 and hMSH2 in epithelial ovarian cancer. Aust N Z J Obstet Gynaecol 2008, 48:505-509.

14. Balch C, Huang TH, Brown R, Nephew KP: The epigenetics of ovarian cancer drug resistance and resensitization. Am J Obstet Gynecol 2004, 191:1552-1572

15. Tamura G: Hypermethylation of tumor suppressor and tumor-related genes in neoplastic and non-neoplastic gastric epithelia. World J Gastrointest Oncol 2009, 1:41-46.

16. Skonier J, Neubauer M, Madisen L, Bennett K, Plowman GD, Purchio AF: cDNA cloning and sequence analysis of beta ig-h3, a novel gene induced in a human adenocarcinoma cell line after treatment with transforming growth factor-beta. DNA Cell Biol 1992, 11:511-522.

17. Zhao $Y L$, Piao CQ, Hei TK: Downregulation of Betaig-h3 gene is causally linked to tumorigenic phenotype in asbestos treated immortalized human bronchial epithelial cells. Oncogene 2002, 21:7471-7477.

18. Shao G, Berenguer J, Borczuk AC, Powell CA, Hei TK, Zhao Y: Epigenetic inactivation of Betaig-h3 gene in human cancer cells. Cancer Res 2006, 66:4566-4573.

19. Ahmed $A A$, Mills $A D$, Ibrahim $A E$, et al: The extracellular matrix protein TGFBI induces microtubule stabilization and sensitizes ovarian cancers to paclitaxel. Cancer Cell 2007, 12:514-527.

20. Shah JN, Shao G, Hei TK, Zhao Y: Methylation screening of the TGFB promoter in human lung and prostate cancer by methylation-specific PCR. BMC Cancer 2008, 8:284.

21. Irigoyen M, Pajares MJ, Agorreta J, et al: TGFBI expression is associated with a better response to chemotherapy in NSCLC. Mol Cancer 2010, 9:130.

22. Ying J, Srivastava G, Hsieh WS, et al: The stress-responsive gene GADD45G is a functional tumor suppressor, with its response to environmental stresses frequently disrupted epigenetically in multiple tumors. Clin Cancer Res 2005, 11:6442-6449.

23. Kang S, Dong SM, Park NH: Frequent promoter hypermethylation of TGFBI in epithelial ovarian cancer. Gynecologic oncology 2010, 118:58-63.

24. Staub J, Chien J, Pan Y, et al: Epigenetic silencing of HSulf-1 in ovarian cancer:implications in chemoresistance. Oncogene 2007, 26:4969-4978.

25. Zhang X, Yashiro M, Ren J, Hirakawa K: Histone deacetylase inhibitor, trichostatin A, increases the chemosensitivity of anticancer drugs in gastric cancer cell lines. Oncol Rep 2006, 16:563-568.

26. Olopade OI, Wei M: FANCF methylation contributes to chemoselectivity in ovarian cancer. Cancer Cell 2003, 3:417-420.

27. Li M, Balch C, Montgomery JS, et al: Integrated analysis of DNA methylation and gene expression reveals specific signaling pathways associated with platinum resistance in ovarian cancer. BMC Med Genomics 2009, 2:34.

\section{doi:10.1186/1756-9966-31-6}

Cite this article as: Wang et al:: TGFBI promoter hypermethylation correlating with paclitaxel chemoresistance in ovarian cancer. Journal of Experimental \& Clinical Cancer Research 2012 31:6. 